\section{Avaliação do índice de comorbidade de Charlson em internações da região de Ribeirão Preto, São Paulo, Brasil}

\author{
Evaluation of the Charlson comorbidity index \\ among inpatients in Ribeirão Preto, São Paulo \\ State, Brazil
}

\author{
1 Escola Nacional de Saúde \\ Pública Sergio Arouca, \\ Fundação Oswaldo Cruz, \\ Rio de Janeiro, Brasil. \\ 2 Faculté de Médecine, \\ Université de Montréal, \\ Montréal, Canada. \\ ${ }^{3}$ Escola Politécnica de Saúde \\ Joaquim Venâncio, Fundação \\ Oswaldo Cruz, Rio de Janeiro, \\ Brasil. \\ Correspondência \\ M. Martins \\ Departamento de \\ Administração e \\ Planejamento em Saúde, \\ Escola Nacional de Saúde \\ Pública Sergio Arouca, \\ Fundação Oswaldo Cruz. \\ Rua Leopoldo Bulhões 1480, \\ Rio de Janeiro, $R J$ \\ 21041-210, Brasil. \\ martins@ensp.fiocruz.br
}

\begin{abstract}
The objective of this article was to evaluate the use of the Charlson comorbidity index (CCI) to predict inpatient death in Ribeirão Preto, São Paulo State, Brazil. 54,680 hospitalizations from January 1996 to December of 1997 were analyzed. Two International Classification of Diseases adaptations of CCI were compared, and the 30 clinical conditions assessed by the Charlson index were reviewed. Logistic regression was used to evaluate the models' capacity to predict inpatient death. The baseline model included: age, sex, and principal diagnosis. Differences in ICD adaptations showed little effect on the models' discriminatory capacity. Revision of the 30 clinical conditions increased the model's discriminatory capacity to predict death (C statistic $=0.73$ ), as compared to the model with the original CCI (C statistic $=0.72)$. All tested models had a reduced effect on the baseline model's discriminatory capacity $(C$ statistic $=0.70)$. The results show the importance of Brazil having an information system that allows a complete description of hospital morbidity in order to monitor health service performance.
\end{abstract}

Hospital Mortality; Comorbidity; Clinical Diagnosis
Mônica Martins 1

Régis Blais 2

Nair Navarro de Miranda 3

\section{Introdução}

O perfil e a gravidade dos casos tratados é um importante fator de confusão para avaliar o desempenho hospitalar 1. Portanto, a mensuração da gravidade representa um importante desafio metodológico. Diversos métodos para medir a gravidade dos casos foram desenvolvidos 2 . Alguns desses podem ser utilizados com informação proveniente de banco de dados administrativos, que dispõem em geral somente de informação diagnóstica. Com essas características o índice de comorbidade de Charlson (ICC) 3 tem sido usado para ajustar indicadores de desempenho por risco, com o intuito de permitir a comparação entre prestadores $4,5,6,7,8$. Contudo, a qualidade $\mathrm{e}$ o valor desse tipo de sistema de classificação de pacientes depende da completitude e precisão dos códigos diagnósticos.

O ICC é composto por vinte condições clínicas selecionadas empiricamente com base no efeito sobre o prognóstico de pacientes internados num serviço de medicina geral dos Estados Unidos. Para construir um índice de comorbidade que discriminasse o prognóstico de paciente em termos da mortalidade no período de até um ano, Charlson et al. 3 avaliaram trinta condições clínicas presentes em uma coorte de 604 casos revistos no New York Hospital (Nova York, Estados Unidos). Com base no risco relativo, vinte condições clínicas foram selecionadas para compor o ICC por apresentarem um risco relativo derivado 
de análise de sobrevida (proportional hazards model) maior que 1,2 (Tabela 1). Nessa metodologia, o risco relativo arredondado foi utilizado para criar uma pontuação. Posteriormente, os resultados encontrados foram validados pelos autores ${ }^{9}$. A aplicação desse índice em banco de dados administrativos se apóia na busca das condições clínicas entre diagnósticos registrados.

As condições clínicas do ICC não foram traduzidas para os códigos da Classificação Internacional de Doenças (CID) pelos autores, adaptações foram desenvolvidas em outros estudos 5,10. O debate sobre a qualidade das adaptações do ICC para a CID indica que apesar das diferenças nos códigos selecionados nas adaptações disponíveis 5,10,11, estas mostraram efeito reduzido sobre o poder de predição do risco de morrer 12,13. Contudo, estes estudos se detiveram na comparação de adaptações baseadas na CID com modificação clínica utilizada na América do Norte 2 . No momento de desenvolvimento do presente estudo, a única adaptação para a Classificação Internacional de Doenças, Lesões e Causas de Óbitos - 9a Revisão (CID-9) 14, usada no Brasil até 1997, disponível na literatura era a realizada por D’Hoore et al. 6. A aplicação dessa adaptação em modelos de predição de óbitos hospitalares apresentou bom desempenho (estatística $\mathrm{C}=0,87 \mathrm{e}$ $\mathrm{C}=0,86) 6,7$.

Alguns estudos questionam a validade dos pesos atribuídos às condições clínicas quando aplicados em pacientes, por exemplo, admitidos em serviços de emergências ou para procedimento eletivo 12,13,15,16. D'Hoore et al. 7 mostraram um bom desempenho do ICC quando aplicado em doenças isquêmicas do coração. O estudo de Ghali et al. 12 não descreveu um desempenho da mesma magnitude e revisaram os pesos do ICC para revascularização do miocárdio. Polanczyk et al. 15 observaram melhores resultados quando utilizaram um índice desenvolvido especificamente para a insuficiência cardíaca congestiva. Essa evidência sobre melhor desempenho de índices desenvolvidos para uma doença específica desdobrou-se em revisões dos pesos do ICC 4,8, entretanto não há relatos sobre a revisão dos pesos atribuídos as trinta condições clínicas originalmente examinadas por Charlson et al. ${ }^{3}$ e que foram excluídas do ICC.

Como conseqüência do desenvolvimento empírico, pode haver no ICC omissões importantes dentre as comorbidades examinadas ou a exclusão de condições clínicas com impacto numa população diferente, além de possível inadequação dos pesos atribuídos às condições clínicas do ICC 9,14. Considerando-se a possibilidade de exclusão de comorbidades relevantes e as limitações de informação diagnóstica nos sistemas de produção hospitalar brasileiros 17, o objetivo deste artigo foi avaliar o uso do ICC para predizer óbito hospitalar em internações na região de Ribeirão Preto, São Paulo, Brasil. Esta avaliação incluiu: (i) adaptação para a CID-9 das trinta condições clínicas originalmente examinadas por Charlson et al. 3; (ii) comparação desta adaptação do ICC para a CID-9 com a desenvolvida por D'Hoore et al. 6; e (iii) revisão da relevância e dos pesos das trinta condições clínicas originariamente examinadas por Charlson et al. 3; (iv) comparação do efeito dos índices de comorbidade, construídos nas etapas anteriores, sobre o desempenho de modelos de predição de óbito hospitalar.

\section{Material e métodos}

\section{Fonte de dados}

Os dados são provenientes do sistema de informação sobre produção hospitalar desenvolvido pela Faculdade de Medicina de Ribeirão Preto, Universidade de São Paulo (FMRP/USP). Esse sistema de informações, concebido nos anos 70, é mantido pelo Centro de Processamento de Dados Hospitalares (CPDH) do Departamento de Medicina Social da FMRP/USP 18. Esse sistema utilizado primordialmente para fins de ensino e pesquisa, é também empregado para a melhoria do cuidado prestado e a administração hospitalar 19,20. A base de dados cobre todas as hospitalizações realizadas na região de Ribeirão Preto, abrangendo o próprio Município de Ribeirão Preto e 19 municípios próximos componentes da região ${ }^{18}$. No sumário de alta, que alimenta essa base de dados, há espaço para o preenchimento de até dois diagnósticos secundários, sendo que a partir de 1998 houve um aumento de mais dois campos para o registro deste tipo de informação. As informações estão disponíveis no nível individual, entretanto não há informação que identifique os pacientes.

Até este momento, nenhum estudo foi publicado sobre a qualidade dos dados do sistema de informação da FMRP/USP. Não obstante, os dados são sistematicamente revisados e atividades de educação continuada são oferecidas aos responsáveis pela coleta de dados nos prontuários médicos e pelo preenchimento dos resumos de alta. Durante o desenvolvimento deste estudo, foram executadas verificações sobre diversos aspectos da qualidade dos dados registrados em 1996 e 1997. Tal verificação incluiu análise da distribuição de freqüência de cada variável do banco de dados, análise bivariada e análises da adequação do processo de seleção dos diagnósti- 
cos principal e secundário. Apesar das limitações, esta análise mostrou que não havia nenhum problema de qualidade significativo na base de dados, e a qualidade dos dados era semelhante entre o público e hospitais privados.

\section{Universo de estudo}

O universo de estudo está circunscrito às hospitalizações realizadas na região de Ribeirão Preto no período entre janeiro de 1996 e dezembro de 1997. Os códigos diagnósticos dos capítulos das doenças dos aparelhos respiratório e circulatório da CID-9 foram selecionados por representar casos com maior probabilidade de ocorrência de óbito, permitindo que este evento possa ser utilizado como medida de resultado. Levando em conta este critério, selecionou-se 54.680 hospitalizações ocorridas em trinta hospitais. Internações de pacientes menores de 18 anos foram excluídas da análise.

\section{Análise dos dados}

\section{- Adaptação das condições clínicas para a CID-9}

O procedimento de adaptação para a CID-9 foi realizado por um pesquisador com formação médica e em codificação de doenças. Este profissional traduziu as trinta condições clínicas listadas por Charlson et al. 3, baseando-se nos critérios de definição das patologias utilizadas por estes autores.

Optou-se neste estudo por readaptar as condições clínicas para a CID-9 para comparar os resultados com os obtidos na adaptação realizada por D'Hoore et al. 6. Para comparar as duas adaptações e verificar em que medidas os escores obtidos apresentariam diferenças significativas, os pesos propostos por Charlson et al. 3 foram aplicados no cálculo do escore de comorbidade de cada caso estudado, considerando a informação registrada nos dois campos de diagnósticos secundários. O coeficiente de correlação de Pearson e a estatística kappa foram empregados para analisar o efeito das diferenças entre as duas adaptações para o conjunto de condições clínicas. A estatística kappa, adequada para dados categóricos ou nominais 21 , mede a concordância corrigida pela concordância aleatória. Landis \& Koch 22 sugerem parâmetros para avaliar o valor do kappa: valores superiores a 0,85 são considerados excelentes e valores inferiores a 0,40 são considerados insatisfatórios.

\section{- Revisão das trinta condições clinicas}

A revisão das trinta condições clínicas examinadas por Charlson et al. ${ }^{3}$ para desenvolver o ICC seguiu uma abordagem semelhante à utilizada por estes autores que incluiu análise bivariada (teste de qui-quadrado) e multivariada (proportional hazards model - modelo de regressão de Cox 23). A variável dependente foi o óbito. A análise bivarida calculou o risco relativo simples. A regressão de Cox foi empregada para calcular o risco relativo ajustado e revisar os pesos referentes ao risco de morrer associado as trinta condições clínicas. No modelo de Cox foram incluídas todas as condições clínicas independente da significância estatística encontrada na análise bivariada. Esse modelo foi construído sem a inclusão do diagnóstico principal. Essa decisão justificase porque o ICC foi concebido para ser um índice genérico, isto é, independente da patologia principal. Apesar da população de estudo incluir somente doenças respiratórias e circulatórias, consideramos que a variabilidade existente é adequada dada a heterogeneidade das patologias incluídas nesses capítulos da CID com relação à gravidade dos casos. As condições clínicas foram avaliadas com relação à sua associação com o óbito, adotando-se como critério de significância estatística valor de $\mathrm{p} \leq 0,10$. O risco relativo ajustado (utilizando as comorbidades como varáveis independes no modelo de Cox) foi utilizado para atribuir pesos para cada condição clínica, em que riscos relativos menores que 1,2 equivalem a 0 ; riscos relativos entre 1,2 e 1,5 equivalem a 1 ; riscos relativos entre 1,5 e 2,5 equivalem a 2 ; e assim em seqüência.

Com o objetivo de comparar o efeito das técnicas estatísticas, a regressão logística também foi utilizada para produzir os pesos das trinta condições clínicas. Nessa etapa, os procedimentos adotados foram os mesmos descritos acima para a regressão de Cox.

\section{- Modelos de predição de óbito}

A regressão logística foi também utilizada para avaliar o impacto dos índices de comorbidade na estimava da chance de morrer no hospital, assim a variável dependente é óbito (sim ou não). Na regressão logística, os índices foram avaliados com base na comparação dos seguintes modelos: (i) usando-se o ICC com a adaptação da CID-9 de D'Hoore et al. 6; (ii) usando-se o ICC com a adaptação para a CID-9 desenvolvida no presente estudo; (iii) usando-se o resultado da revisão das trinta condições clínicas utilizando-se a regressão de Cox para gerar novos pesos; e (iv) usando-se a revisão das trinta condições clínicas 
utilizando-se a regressão logística para gerar novos pesos.

Considerando-se os fatores de risco relevantes e as informações disponíveis na base de dados, o modelo de base criado incluiu as seguintes variáveis: idade, sexo e diagnóstico principal. A idade foi tratada como variável contínua. O sexo masculino foi a categoria de referência. Diagnósticos principais específicos foram selecionados de acordo com critérios de volume de casos e taxa de mortalidade superior a 4,9\%. Os códigos diagnósticos selecionadosforam: pneumonias (CID-9: 481, 482, 485, 486), insuficiência cardíaca (CID-9: 428), doença isquêmica do coração (CID-9: $410,411,413,414)$ e doença cerebrovascular (CID-9: 430-434 436,438). A variável diagnóstico principal foi tratada com uma variável categórica, cuja categoria de referência foi pneumonia pneumocócica (CID-9: 481) por apresentar a taxa de mortalidade menos elevada. No modelo de base foi introduzido separadamente cada um dos escores de comorbidade calculado, estes foram empregados como variável contínua. Neste estudo a validade interna dos modelos foi avaliada com base no nível de discriminação e calibração. A capacidade de cada um dos escores de melhorar a predição de óbito foi avaliada pela estatística C (medida de discriminação), e o teste de Hosmer-Lemeshow (medida de calibração) foi empregado para avaliar problemas no ajuste dos modelos 2,24. As análises estatísticas foram processadas no programa SPSS (SPSS Inc., Chicago, Estados Unidos).

\section{Resultados}

Na Tabela 1 é apresentado o resultado da adaptação das condições clínicas que compõem o ICC para a CID-9 realizada por D'Hoore et al. 6 e neste estudo. Na comparação entre essas adaptações, observam-se diferenças na codificação das seguintes condições clínicas: infarto do miocárdio, insuficiência cardíaca congestiva, doença cerebrovascular, doença crônica do fígado e cirrose, doença pulmonar crônica, diabetes e hemiplegia. Por exemplo, no infarto do miocárdio optouse por incluir outros códigos do subgrupo: doenças isquêmicas do coração, excluindo somente o código para angina (CID-9: 413). D’Hoore et al. 6 restringiram-se ao infarto agudo do miocárdio (CID-9: 410) e outras formas agudas e subagudas da doença isquêmica do coração (CID-9: 411). Para o diabetes, Charlson et al. 3 distinguem os casos segundo o critério da existência ou não de lesão de órgão. D’Hoore et al. 6 não fizeram qualquer distinção devido a presença de complicações. No presente estudo optou-se por separar os casos de diabetes simplesmente entre aqueles com complicação daqueles sem complicação (Tabela 1).

No período entre 1996 e 1997 ocorreram 54.680 internações devido a problemas cardiovasculares e respiratórios. A região de Ribeirão Preto abrange 19 municípios, no período estudado 39\% das internações foram de residentes do próprio Município de Ribeirão Preto. A idade média dos pacientes era de 57 anos e o percentual de internações entre homens e mulheres é semelhante (Tabela 2).

As internações foram preponderantemente financiadas pelo SUS e ocorreram em hospitais privados. Pacientes permaneceram em média internados cinco dias e procedimentos cirúrgicos foram realizados em $24 \%$ dos casos. Mais de $90 \%$ dos casos tiveram alta, os óbitos corresponderam a 7,1\% (Tabela 2).

Em 1996 e 1997, as internações com anotação de diagnóstico secundário corresponderam a $42,6 \%$, dos quais cerca de $17 \%$ apresentaram um segundo diagnóstico (Tabela 2). Observou-se maior proporção de óbitos nos casos que registraram dois diagnósticos secundários. A taxa de mortalidade é crescente em função do número de diagnósticos secundários (comorbidades); pacientes sem comorbidade tiveram taxa de mortalidade de $4 \%$, pacientes com uma comorbidade tiveram taxas de mortalidade de $7 \%$ e pacientes com duas comorbidades tiveram taxas de mortalidade de $18 \%$ ( $\chi^{2}: 2085,86$, df 2 , sig 0,000 ). A razão de chance (odds ratio - OR) entre presença de comorbidades e óbito foi alta (OR: 3,03; IC95\%: 2,83-3,25).

As diferenças nas traduções para a CID-9 descritas acima resultaram um maior percentual de casos com escore igual a 0 quando se aplica a adaptação de D'Hoore et al. 6 (85,4\%), contra $80,8 \%$ quando se aplica a adaptação do presente estudo. Também se observou uma maior taxa de mortalidade nesta categoria, $6 \%$ em comparação com 5,6\% quando se utiliza a adaptação feita para este trabalho. Entretanto, o somatório global dos escores é semelhante (13.473 vs. 13.471) quando os casos de AIDS são excluídos, uma vez que D’Hoore et al. ${ }^{6}$ não se ateve a esta patologia. Os valores preditos pelas duas traduções apresentaram alta correlação de Pearson $(0,96 ; p<0,000)$. A concordância simples entre as duas traduções foi de 89,8 e a estatística Kappa de 0,67 (SE: 0,004).

A Tabela 3 apresenta os resultados das análises bivariada e multivariada das trinta condições clínicas examinadas por Charlson et al. ${ }^{3}$ para o desenvolvimento do ICC e os pesos calculados com base nos modelos de regressão de Cox e logística. As condições clínicas com riscos relativos simples e ajustados significativos foram: angina, 
Índice de comorbidade de Charlson (ICC): adaptação das condições clínicas para a Classificação Internacional de Doenças, Lesões e Causas de Óbitos 9ạ Revisão (CID-9)

\begin{tabular}{|c|c|c|c|}
\hline Peso do ICC & Condição clínica (ICC) & CID-9 (Pesquisa) * & CID-9 (D’Hoore et al.) ** \\
\hline \multirow[t]{10}{*}{1} & Infarto do miocárdio & $410-412,414$ & 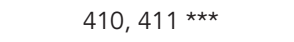 \\
\hline & Insuficiência cardíaca congestiva & 402,428 & $398,402,428$ \\
\hline & Doença vascular periférica & $440-447$ & $440-447$ \\
\hline & Doença cérebro-vascular & $430-438$ & 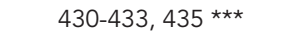 \\
\hline & Demência & $290,291,294$ & $290,291,294$ \\
\hline & Doença pulmonar crônica & $490-496$ & $491-493$ \\
\hline & Doença do tecido conjuntivo & $710,714,725$ & $710,714,725$ \\
\hline & Úlcera & $531-534$ & $531-534$ \\
\hline & Doença crônica do fígado e cirrose & $571,573,578$ & $571,573 * \star \star$ \\
\hline & Diabetes sem complicação & 250.0 & $\star \star \star$ \\
\hline \multirow[t]{6}{*}{2} & Hemiplegia ou paraplegia & 342,344 & 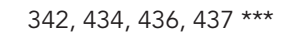 \\
\hline & Doença renal severa ou moderada & $403,404,580-586$ & $403,404,580-586$ \\
\hline & Diabetes com complicação & $250(1-9)$ & $250 * \star \star$ \\
\hline & Tumor & $140-195$ & $140-195$ \\
\hline & Leucemia & 204-208 & $204-208$ \\
\hline & Linfoma & $200,202,203$ & $200,202,203$ \\
\hline 3 & Doença do fígado severa ou moderada & $070,570,572,456.0,456.2$ & $070,570,572$ \\
\hline \multirow[t]{2}{*}{6} & Tumor maligno, metástase & 196-199 & 196-199 \\
\hline & AIDS & 279.1 & $\star \star \star$ \\
\hline
\end{tabular}

* Tradução das condições clínicas propostas por Charlson et al. 3 para a CID-9 realizada pela equipe da pesquisa;

** Tradução das condições clínicas propostas por Charlson et al. 3 para a CID-9 realizada por D'Hoore et al. 6;

*** Indica as diferenças entre os códigos diagnósticos utilizados nas duas adaptações das condições clínicas.

arritmia, infarto de miocárdio, insuficiência cardíaca congestiva, doença vascular periférica, doença cerebrovascular, doença pulmonar, outras doenças endócrinas, renal grave e moderada, doenças crônicas do fígado e cirrose, hemorragia gastrintestinal, tumor, leucemia, AIDS, metástase e coagulopatias (Tabela 3 ). Em geral, os pesos obtidos com a regressão logística foram comparativamente maiores que os obtidos com a regressão de Cox. Divergências quanto à significância das condições clínicas foram observadas. Doença valvular, outras doenças neurológicas, diabetes, insuficiência renal leve e linfoma mostraram significância estatística quando a regressão logística foi utilizada para o cálculo dos pesos (Tabela 3). Contudo, no caso da diabetes o peso atribuído foi o mesmo, isto é, escore igual a 0 .

A aplicação das duas adaptações da CID-9 em modelos de predição de óbito apresentaram bom desempenho, tanto com respeito ao ajuste dos modelos quanto à capacidade de discriminação (Tabela 4). Observa-se uma ligeira superioridade na tradução desenvolvida nesta pesquisa quanto à capacidade de discriminação (modelo 3, estatística $\mathrm{C}=0,72$ vs. modelo 2, estatística $\mathrm{C}=0,71$ ).
A revisão do peso das trinta condições clínicas melhorou o modelo de predição de óbito quando comparado ao uso do ICC (modelo 3, estatística $\mathrm{C}=0,72$; modelo 4, estatística $\mathrm{C}=0,73$ ) (Tabela 4). Não foram observadas melhorias importantes na capacidade preditiva (estatística C) dos modelos, advindas do uso da regressão logística para o cálculo dos pesos comparativamente ao uso da regressão de Cox, a estatística C variou em 0,004 quando compara-se às técnicas estatísticas. O teste de Hosmer-Lemeshow não mostrou problema de ajuste nos modelos testados.

\section{Discussão}

A adaptação para a CID-9 realizada neste estudo apresenta diferenças com relação aos códigos diagnósticos empregados por D’Hoore et al. 6, gerando um kappa moderado 22 . Esse valor se explica, sobretudo pelos códigos e distinção utilizada para o diabetes. Contudo, os resultados encontrados mostraram que diferenças na tradução para a CID-9 não tiveram efeito significativo sobre o escore de gravidade dos pacientes e a capacidade discriminativa dos modelos de predição 
Características da população estudada.

Caracteristicas

Número de casos

Demográficas

Idade média (anos)

Homem (\%)

Habitantes de Ribeirão Preto (\% internações)

Procedimento cirúrgico (\%)

Comorbidade (\%)

Registro de um diagnóstico secundário

Registro de dois diagnósticos secundários

Casos sem diagnóstico secundário

Tempo de permanência (dias)

Média

Mediana

Resultado do cuidado (\%)

Alta

Transferência

Óbito

Fonte de pagamento (\%)

SUS

Seguro-saúde

Particular

Natureza jurídica do hospital (\%)

Público

Privado

Diagnóstico principal (capítulos da CID-9) (\%)

Aparelho circulatório

Aparelho respiratório

Internações (1996-1997)

54.680

57,1 (DP 18,4)

49,8

38,8

24,3

25,9

16,7

57,4

4,8

91,9

0,8

7,1

52,0

44,3

3,7

19,6

80,4

63,1

36,9

SUS: Sistema Único de Saúde; CID-9: Classificação Internacional de Doenças, Lesões e Causas de Óbitos - 9ạ Revisão.

de óbito. Essas conclusões estão em acordo com as relatadas na literatura; apesar das diferenças entre os códigos diagnósticos empregados 5,10, a aplicação de diferentes adaptações em modelos de predição de óbito mostraram ganho reduzido sobre a capacidade de discriminação dos mode$\operatorname{los} 12,13$.

A revisão das trinta condições clínicas estudadas por Charlson et al. 3 , e de seus pesos, aumentou a capacidade de discriminação dos modelos de predição de óbito quando comparados ao modelo com o ICC original. Esse resultado sublinha a importância de refazer o percurso metodológico desenhado por Charlson et al. 3,9 .

Contudo, todos os modelos testados tiveram efeito reduzido sobre a capacidade preditiva do modelo de base (estatística $\mathrm{C}=0,70$ ). Esse resultado é semelhante ao encontrado em outro estudo que utilizou o Sistema de Informações Hospitalares do Sistema Único da Saúde (SIH-SUS) 17, no qual idade e diagnóstico principal foram os principais preditores de óbito. Comparando-se o uso do ICC aplicado ao SIH-SUS 17 e ao sistema de informação de Ribeirão Preto, a capacidade preditiva obtida neste ultimo é maior (estatística C do SIH-SUS $=0,68 v s$. estatística C de Ribeirão Preto $=0,71$ ). Além disso, no presente estudo foi encontrada uma OR de 3,03 entre presença de comorbidades e óbito. Esse achado indica a importância de dispor de maior número de campos para o registro das comorbidades de pacientes hospitalizados no Brasil. Até o momento não existe no país um sistema de informação que permita uma descrição completa da morbidade hospitalar, o que limita, entre outras, a utilidade dessas informações para a avaliação e o monitoramento do desempenho dos serviços.

Com relação às técnicas estatísticas utilizadas para gerar pesos para as condições clínicas do ICC, alguns autores empregaram a regressão logística e outros a análise de sobrevida (modelo de Cox) 3,12,15,16. A justificativa para o uso da re- 
Risco relativo e razão de chance entre óbito e condições clínicas do índice de comorbidade de Charlson (ICC).

\begin{tabular}{|c|c|c|c|c|c|c|c|c|c|}
\hline Condição clínica & Casos & Mortes (\%) & $R R$ * $(p)$ & $\begin{array}{c}\text { RR ajustado ** } \\
\text { (p) }\end{array}$ & Pesos RR *** & OR \# & Peso & OR & $\# \#$ \\
\hline \multicolumn{10}{|l|}{ Miocárdio } \\
\hline Angina & 263 & 3,0 & $0,40(0,010)$ & $0,33(0,002)$ & 0 & 0,44 & & 0 & \\
\hline Arritmias & 1.015 & 13,2 & $2,02(0,000)$ & $1,67(0,000)$ & 2 & 2,08 & & 2 & \\
\hline Valvular & 346 & 9,8 & $1,42(0,050)$ & $0,92(0,643) \# \#$ & 0 & 1,42 & & 1 & \\
\hline Infarto do miocárdio & 1.205 & 16,5 & $2,66(0,000)$ & $1,81(0,000)$ & 2 & 2,74 & & 3 & \\
\hline Insuficiencia cardíaca congestiva & 1.969 & 11,0 & $1,65(0,000)$ & $1,43(0,000)$ & 1 & 1,71 & & 2 & \\
\hline \multicolumn{10}{|l|}{ Vascular } \\
\hline Hipertensão & 3.713 & 7,1 & $1,00(0,984) \S$ & $0,91(0,157)$ \#\#\# & 0 & 1,01 \#\#\# & & 0 & \\
\hline Vascular periférica & 474 & 33,3 & $6,75(0,000)$ & $3,03(0,000)$ & 3 & 6,52 & & 7 & \\
\hline Cerebrovascular & 1.163 & 26,0 & $4,87(0,000)$ & $2,73(0,000)$ & 3 & 5,16 & & 5 & \\
\hline \multicolumn{10}{|l|}{ Pulmonar } \\
\hline Crônica & 2.320 & 9,3 & $1,35(0,000)$ & $1,30(0,000)$ & 1 & 1,49 & & 1 & \\
\hline Grave e moderada & 507 & 28,4 & $5,33(0,000)$ & $3,06(0,000)$ & 3 & 5,85 & & 6 & \\
\hline \multicolumn{10}{|l|}{ Neurológica } \\
\hline Outra neurológica & 140 & 13,6 & $2,05(0,003)$ & $1,37(0,177) \# \# \#$ & 1 & 2,13 & & 2 & \\
\hline Demência & 64 & 12,5 & $1,86(0,095)$ & $1,45(0,297) \# \# \#$ & 1 & 1,91 \#\#\# & & 2 & \\
\hline Hemiplegia e paraplegia & 32 & 9,4 & $1,35(0,621) \S$ & $1,02(0,976) \# \# \#$ & 1 & $1,39 \# \#$ & & 1 & \\
\hline \multicolumn{10}{|l|}{ Endócrina } \\
\hline Outra endócrina & 13 & 23,1 & $3,91(0,025)$ & $5,10(0,005)$ & 5 & 4,41 & & 4 & \\
\hline Diabetes & 2.553 & 8,0 & $1,13(0,098)$ & $1,04(0,626) \# \# \#$ & 0 & 1,16 & & 0 & \\
\hline Diabetes com complicação & 104 & 8,7 & $1,24(0,545) \S$ & $0,72(0,322) \# \# \#$ & 0 & 1,11 \#\#\# & & 0 & \\
\hline \multicolumn{10}{|l|}{ Renal } \\
\hline Insuficiência renal leve & 151 & 13,2 & $1,99(0,003)$ & $0,97(0,903) \# \# \#$ & 0 & 1,82 & & 2 & \\
\hline Grave e moderada & 510 & 25,1 & $4,48(0,000)$ & $2,80(0,000)$ & 3 & 4,79 & & 5 & \\
\hline \multicolumn{10}{|l|}{ Fígado } \\
\hline Crônica e cirroses & 263 & 22,1 & $3,73(0,000)$ & $2,26(0,000)$ & 2 & 3,73 & & 4 & \\
\hline Grave e moderada & 74 & 17,6 & $2,78(0,000)$ & $1,20(0,520) \# \# \#$ & 1 & 1,63 \#\#\# & & 2 & \\
\hline \multicolumn{10}{|l|}{ Gastro-intestinal } \\
\hline Hemorragia digestiva & 101 & 28,7 & $5,28(0,000)$ & $2,53(0,000)$ & 2 & 5,14 & & 5 & \\
\hline Inflamação do intestino & 2 & 0,0 & $1,00(0,695) \S$ & $0,00(0,899) \# \# \#$ & 0 & 0,04 \#\#\# & & 0 & \\
\hline Úlcera & 101 & 5,0 & $0,68(0,394) \S$ & $0,56(0,190) \# \# \#$ & 0 & $0,67 \# \# \#$ & & 0 & \\
\hline \multicolumn{10}{|l|}{ Câncer/Imunitária } \\
\hline Tumor & 309 & 25,6 & $4,55(0,000)$ & $2,39(0,000)$ & 2 & 4,46 & & 5 & \\
\hline Limfomas & 58 & 19,0 & $3,06(0,000)$ & $1,53(0,159) \# \#$ & 1 & 3,24 & & 3 & \\
\hline Leucemia & 42 & 28,6 & $5,22(0,000)$ & $2,46(0,002)$ & 2 & 6,63 & & 7 & \\
\hline AIDS & 266 & 26,7 & $4,81(0,000)$ & $2,60(0,000)$ & 3 & 5,62 & & 6 & \\
\hline Metástase & 61 & 34,4 & $6,87(0,000)$ & $2,68(0,000)$ & 3 & 4,05 & & 4 & \\
\hline \multicolumn{10}{|l|}{ Outras } \\
\hline Reumatológicas & 125 & 7,2 & $1,01(0,975) \S$ & $0,58(0,105) \# \# \#$ & 0 & $0,79 \# \# \#$ & & 0 & \\
\hline Coagulopatias & 23 & 21,7 & $3,62(0,006)$ & $3,22(0,009)$ & 3 & 4,39 & & 4 & \\
\hline
\end{tabular}

$\mathrm{RR}=$ risco relativo; $\mathrm{OR}=$ odds ratio.

* Análise bivariada;

** Modelo de regressão de Cox;

*** Peso derivado do risco relativo ajustado pela regressão de $\operatorname{Cox}(\mathrm{RR}<1,2$ peso igual a 0$)$;

\# Modelo de regressão logística;

\#\# Peso derivado do risco relativo ajustado pela regressão logística ( $O R<1,2$ peso igual a 0);

\#\#\# Não significativo, $\mathrm{p}>0,10$;

$\S$ Não significativo, $\mathrm{p}>0,15$. 
Tabela 4

Capacidade de discriminação e ajuste dos modelos de predição de óbito hospitalar.

\begin{tabular}{|c|c|c|c|}
\hline Modelo & $\chi^{2}$ do modelo & $\begin{array}{c}\text { Teste } \chi^{2} \text { de Hosmer } \\
\& \text { Lemeshow }\end{array}$ & $\begin{array}{l}\text { Estatística C } \\
\text { (IC95\%) }\end{array}$ \\
\hline \multirow[t]{2}{*}{ 1. Modelo de base: diagnóstico principal, idade e sexo } & 1110,13 & $8,9(p=0,351)$ & $0,70(0,69-0,71)$ \\
\hline & $(g \mid 15, p=0,000)$ & & \\
\hline \multirow[t]{2}{*}{ 2. Modelo de base e ICC tradução para CID-9 de D’Hoore et al. 6} & 1276,11 & $5,15(p=0,742)$ & $0,71(0,70-0,72)$ \\
\hline & $(g \mid 16, p=0,000)$ & & \\
\hline \multirow[t]{2}{*}{ 3. Modelo de base e ICC tradução para CID-9 do presente estudo } & 1394,65 & $10,50(p=0,232)$ & $0,72(0,71-0,73)$ \\
\hline & $(g \mid 16, p=0,000)$ & & \\
\hline \multirow[t]{2}{*}{ 4. Modelo de base e trinta condições clínicas de Charlson (regressão de Cox) } & 1665,81 & $8,3(p=0,405)$ & $0,73(0,72-0,74)$ \\
\hline & $(g \mid 16, p=0,000)$ & & \\
\hline \multirow[t]{2}{*}{ 5. Modelo de base e trinta condições clínicas de Charlson (regressão logística) } & 1732,51 & $9,6(p=0,294)$ & $0,74(0,73-0,75)$ \\
\hline & $(g \mid 16, p=0,000)$ & & \\
\hline
\end{tabular}

ICC = índice de comorbidade de Charlson; CID-9: Classificação Internacional de Doenças, Lesões e Causas de Óbitos - 9ạ Revisão.

gressão logística está relacionada ao fato de que o evento a ser predito (óbito hospitalar) ocorre num período curto de tempo. As duas técnicas estatísticas foram testadas neste estudo; apesar das diferenças nos pesos das condições clínicas, estas não representam melhorias importantes na capacidade de predição dos modelos (estatística C) quando se recorreu à regressão logística para calcular os pesos comparativamente à regressão logística. Além disso, a regressão de Cox apresenta como vantagem a correção com relação ao tempo de observação do evento estudado e a censura dos dados 23. Por essas características, o modelo de Cox permite tratar de forma mais adequada a relação entre o tempo de permanência e a mortalidade hospitalar, que influencia a probabilidade da ocorrência de mortes após a alta hospitalar relacionadas ao cuidado anteriormente recebido, mas que ocorrem. Estudos sugerem que diferenças na mortalidade hospitalar são resultado de diferenças na permanência dos pacientes $25,26,27,28$. Isto é, variações no tempo de permanência devido a diferenças nas políticas de admissão e alta ou no acesso a serviços domiciliares e a cuidados de longa permanência influenciam o lugar onde a morte ocorre. Hospitais com tempo médio de permanência baixo podem ter uma taxa de mortalidade também mais baixa, seja porque seus pacientes são transferidos mais freqüentemente ou porque morrem fora do hospital 27,29.

É importante reconhecer que os resultados encontrados com relação à capacidade preditiva dos modelos com ICC original e com as trinta condições clínicas quando comparados ao modelo de base podem ser devido a diferenças entre as populações de estudo e a de desenvolvimento do ICC, ou à composição estrutura e limitação dos dados. É importante destacar que a população de estudo está circunscrita às doenças circulatórias e respiratórias, isto pode em parte explicar os pesos obtidos para as seguintes condições clínicas: doença vascular periférica, doença cerebrovascular, doença pulmonar leve e moderada e coagulopatias. Por outro lado, esse resultado pode refletir a importância e a gravidade dessas patologias no perfil de casos de pacientes brasileiros. Por fim, o presente estudo não teve por objetivo discutir a validade do ICC no contexto brasileiro, para tal são necessários outros estudos com desenho mais adequado. O presente estudo representa sim um primeiro passo nessa discussão que envolverá a aplicação desses resultados em outras populações antes de generalizar seu uso no Brasil.

Alguns trabalhos sublinham a importância de gerar empiricamente pesos, particularmente quando se avalia diagnóstico ou procedimento específico, e incluir outras comorbidades no ICC para aumentar o poder de predição de modelos de predição de risco 11,12,13,15,16,30,31,32. Essa estratégia contribui para a criação de índices mais adequados à população de estudo, ao diagnóstico principal e ao resultado do cuidado. Entretanto, o desenvolvimento de um índice e novos pesos em cada estudo dificulta a comparação dos achados, é custoso e fere o princípio científico de parcimônia. Portanto, o ICC permanece útil, mas as bases de dados brasileiras precisam ser enriquecidas com relação à informação diagnóstica disponível. Esse limite está colocado, sobretudo para o SIHSUS, uma vez que o sistema de Ribeirão Preto desde 1998 passou a registrar até quatro diagnósticos secundários. Em outros países, os resumos de alta permitem a codificação de diagnósticos secundários variando entre 15 e 25 campos 2 . 


\section{Resumo}

O objetivo deste artigo foi avaliar o uso do índice de comorbidade de Charlson (ICC) para predizer óbito hospitalar em internações da região de Ribeirão Preto, São Paulo, Brasil. Foram analisadas 54.680 hospitalizações entre janeiro de 1996 e dezembro de 1997. Duas adaptações do ICC para a Classificação Internacional de Doenças (CID) foram comparadas e as trinta condições clínicas avaliadas por Charlson foram revistas. A regressão logística foi utilizada para avaliar a capacidade dos modelos de predizer o óbito hospitalar. O modelo de base incluiu: idade, sexo e diagnóstico principal. Diferenças na adaptação para a CID-9 pouco impactaram a capacidade de discriminação dos modelos. A revisão das trinta condições clínicas aumentou a capacidade de discriminação do modelo de predição de óbito (estatística $C=0,73$ ) quando comparado ao modelo com o ICC original (estatística $C=0,72$ ). Todos os modelos testados tiveram efeito reduzido sobre a capacidade discriminativa do modelo de base (estatística $C=0,70$ ). Os resultados apontam a importância de se dispor no país de um sistema de informação que permita uma descrição completa da morbidade hospitalar para o monitoramento do desempenho dos serviços.

Mortalidade Hospitalar; Comorbidade; Diagnóstico Clínico

\section{Referências}

1. Hornbrook MC. Hospital case mix: its definitions, measurement and use: part I. The conceptual framework. Med Care Rev 1982; 39:1-43.

2. Iezzoni L. Risk adjustment for measuring health care outcomes. 3rd Ed. Ann Arbor: Health Administration Press; 2003.

3. Charlson ME, Pompei P, Ales KL, MacKenzie CR. A new method of classifying prognostic comorbidity in longitudinal studies: development and validation. J Chronic Dis 1987; 40:373-83.

4. Groot V, Becherman H, Lankhorst G, Bouter LM How to measure comorbidity: a critical review of available methods. J Clin Epidemiol 2003; 56: 221-9.

5. Deyo RA, Cherkin DC, Ciol MA. Adapting a clinical comorbidity index for use with ICD-9-CM administrative databases. J Clin Epidemiol 1992; 45: 613-9.

6. D'Hoore W, Sicotte C, Tilquin C. Risk adjustment in outcome assessment: the Charlson comorbidity index. Methods Inf Med 1993; 32:382-7.

\section{Colaboradores}

M. Martins participou de todas as etapas deste estudo. R. Blais participou na elaboração do estudo e discussão dos resultados. N. N. Miranda participou da revisão da definição das condições clínicas e sua adaptação para a Classificação Internacional de Doenças.

\section{Agradecimentos}

W. K. Kellogg Foundation pela bolsa de doutorado e ao Programa de Pesquisa Estratégica da Escola Nacional de Saúde Pública Sergio Arouca, Fundação Oswaldo Cruz, pelo apoio financeiro. Ao Dr. Juan Stuardo Yazle Rocha, coordenador do Centro de Processamento de Dados Hospitalares da Faculdade de Medicina de Ribeirão Preto, Universidade de São Paulo, pelo acesso à base de dados do estudo.
7. D'Hoore W, Bouckaert A, Tilquin C. Practical considerations on the use of the Charlson comorbidity index with administrative data bases. J Clin Epidemiol 1996; 42:1429-33.

8. Needham DM, Scales DC, Laupacis A, Pronovost PJ. A systematic review of the Charlson comorbidity index using administrative databases: a perspective on risk adjustment in critical care research. J Crit Care 2005; 20:12-9.

9. Charlson ME, Szatrowski TP, Peterson J, Gold J. Validation of a combined comorbidity index. J Clin Epidemiol 1994; 47:1245-51.

10. Romano PS, Roos LL, Jollis JG. Adapting a clinical comorbidity index for use with ICD-9-CM administrative data: differing perspectives. J Clin Epidemiol 1993; 46:1075-9.

11. Romano PS, Roos LL, Jollis JG. Further evidence concerning the use of a clinical comorbidity index with ICD-9-CM administrative data. J Clin Epidemiol 1993; 46:1085-90. 
12. Ghali WA, Hall RE, Rosen AK, Ash AS, Moskowitz MA. Searching for an improved clinical comorbidity index for use with ICD-9-CM administrative data. J Clin Epidemiol 1996; 49:273-8.

13. Cleves M, Sanchez N, Draheim M. Evaluation of two competing methods for calculating Charlson Comorbidity Index when analyzing short-term mortality using administrative data. J Clin Epidemiol 1997; 50:903-8.

14. Organização Mundial da Saúde. Manual da classificação estatística internacional de doenças, lesões e causas de óbitos - 9a conferência de revisão. São Paulo: Centro Brasileiro de Classificação de Doenças em Português; 1985.

15. Polanczyk CA, Rohde LE, Philben EA, DiSalso TG. A new casemix adjustment index for hospital mortality among patients with congestive heart failure. Med Care 1998; 36:1489-99.

16. Elixhauser A, Steiner C, Harris DR, Coffey RML. Comorbidity measures for use with administrative data. Med Care 1998; 36:8-27.

17. Martins M, Travassos C, Noronha JC. Sistema de Informações Hospitalares como ajuste de risco de índices de desempenho. Rev Saúde Pública 2001; 35:185-92.

18. Rocha JSY, Simões BJ, Guedes GL. Assistência hospitalar como indicador de desigualdade social. Rev Saúde Pública 1997; 31:479-87.

19. Rocha JSY, Rufino Neto J, Nogueira JL. Quarenta anos da F.M.R.P. - USP - O Departamento de Medicina Social. Medicina (Ribeirão Preto) 1992; 25: 74-84.

20. Rocha JSY, Simões BJ, Forster AC. Estudos de demanda e utilização de serviços de saúde: uma experiência regional. Medicina (Ribeirão Preto) 1993; 26:636-42.

21. Fleiss JL. Statistical methods for rates and proportions. New York: John Wiley and Sons; 1981.

22. Landis JR, Koch GG. The measurement of observer agreement for categorical data. Biometrics 1977; 33:159-74.
23. Hill C, Com-Nougué C, Kramar A, Moureau T, O'Quigley J, Senoussi R, et al. Analyse statistique des données de survie. Paris: Éditions INSERM;1990.

24. Hosmer DW, Lemeshow S. Applied logistic regression. New York: John Wiley and Sons; 1989.

25. Jenks SF, Williams DK, Kay TL. Assessing hospital associated deaths from discharge data: the role pf length of stay and comorbidity. JAMA 1988; 260:2240-6.

26. Kaboli PJ, Barnett MJ, Fuehrer SM, Rosenthal GE. Length of stay as a source of bias in comparing performance in VA and private sector facilities: lessons learned from a regional evaluation of intensive care outcomes. Med Care 2001; 39:1014-24.

27. Thomas JW, Hofer TP. Research evidence on the validity of risk-adjusted mortality rates as a measure of hospital quality of care. Med Care Res Rev 1998; 55:371-404.

28. Kahn KL, Brook RH, Draper D, Keeler EB, Rubenstein LV, Rogers WH, et al. Interpreting hospital mortality data: how can we proceed? JAMA 1988; 260:3625-8.

29. Thomas JW, Guire KE, Horvat GG. Is patient length of stay related to quality of care? Hosp Health Serv Adm 1997; 42:489-506.

30. Stukenborg GJ, Wagner DP, Connors AF. Comparison of the performance of two measures, with and without information from prior hospitalizations. Med Care 2001; 39:727-39.

31. van Doorn C, Bogardus ST, Williams CS, Concato J, Towle VR, Inouye SK. Risk adjustment for older hospitalized persons: a comparison of two methods of data collection for Charlson index. J Clin Epidemiol 2001; 54:694-701.

32. Kieszak SM, Flanders WD, Kosinski AS, Shipp CC, Karp H. A comparison of the Charlson Comorbidity Index derived from medical record data and administrative billing data. J Clin Epidemiol 1999; 52:137-42.

Recebido em 08/Jan/2007

Versão final reapresentada em 29/Jun/2007 Aprovado em 13/Jul/2007 